\title{
Epidemic Information Dissemination in Mobile Molecular Communication Networks for Target Detection Applications
}

\author{
Yutaka Okaie \\ Graduate School of \\ Frontier Biosciences, \\ Osaka University \\ Suita, Japan \\ yokaie@ \\ fbs.osaka-u.ac.jp
}

\author{
Tadashi Nakano \\ Graduate School of \\ Frontier Biosciences, \\ Osaka University \\ Suita, Japan \\ tadasi.nakano@ \\ fbs.osaka-u.ac.jp
}

\author{
Takahiro Hara \\ Graduate School of \\ Information Science and \\ Technology, \\ Osaka University \\ Suita, Japan \\ hara@ \\ ist.osaka-u.ac.jp
}

\begin{abstract}
Epidemic information dissemination is a promising technique for delivering messages in sparse mobile networks. This paper proposes an epidemic information dissemination method for mobile molecular communication networks. We first describe a model of the proposed epidemic information dissemination for mobile molecular communication networks. We then provide numerical experimental results showing the performance of the proposed method in terms of the fraction of bio-nanomachines that successfully receive information. Further, we apply the proposed information dissemination method to a target detection problem in mobile molecular communication networks.
\end{abstract}

\section{CCS Concepts}

-Applied computing $\rightarrow$ Health care information systems; •Hardware $\rightarrow$ Biology-related information processing;

\section{Keywords}

Epidemic information dissemination, bio-nanomachine, mobile molecular communication, target detection, drug delivery

\section{INTRODUCTION}

Epidemic information dissemination enables message delivery among mobile nodes in sparse mobile networks [9]. In analogy with the spreading of infectious diseases, messagecarrying nodes transmit message copies to other nodes when they encounter each other. Namely, these nodes that receive message copies are infected and become message-carrying

Permission to make digital or hard copies of all or part of this work for personal or classroom use is granted without fee provided that copies are not made or distributed for profit or commercial advantage and that copies bear this notice and the full citation on the first page. To copy otherwise, to republish, to post on servers or to redistribute to lists, requires prior specific permission and/or a fee. BICT 2017, March 15-16, Hoboken, United States

ISBN 978-1-63190-148-5

DOI: 10.4108/eai.22-3-2017.152408

Copyright $\odot 2017$ EAI

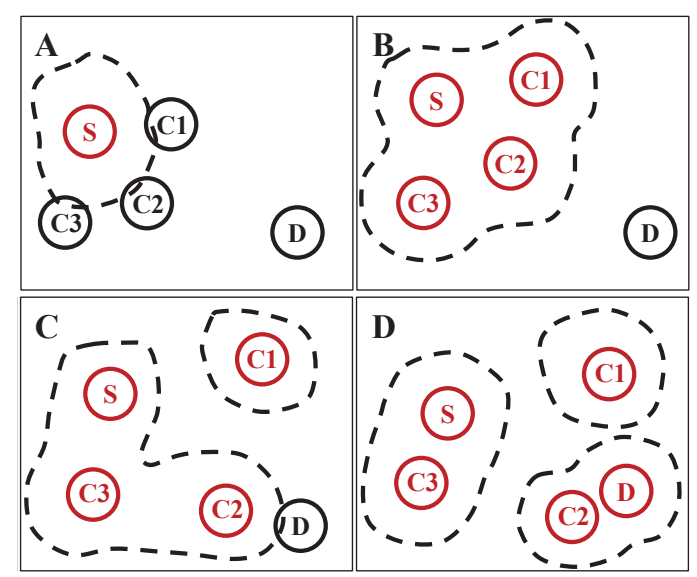

Figure 1: Epidemic information dissemination in mobile molecular communication networks

nodes. By repeating this infectious process, a message is delivered from a source node to the destination node.

Inspired by how messages are delivered through the epidemic information dissemination, this paper proposes a method by which bio-nanomachines migrate and propagate messages in mobile molecular communication networks $[7,8,10]$. In the proposed method (Fig. 1), three types of bio-nanomachine are considered: a source $(\mathrm{S})$, carriers $\left(\mathrm{C}_{1}, C_{2}\right.$ and $\left.\mathrm{C}_{3}\right)$ and a destination (D) [6]. Here $\mathrm{S}$ wishes to transmit a messagecarrying molecule to $\mathrm{D}$, but $\mathrm{D}$ is outside the communication range indicated by the dashed line in (A). The mobile carriers $\left(\mathrm{C}_{1}, C_{2}\right.$ and $\left.\mathrm{C}_{3}\right)$ within the communication range are then leveraged to expand the $\mathrm{S}$ 's communication range as in (B). These carriers receive the message-carrying molecule that $\mathrm{S}$ transmitted and start producing its copies. They also continue to move in the environment and hence dynamically change the S's communication range as in $(\mathrm{C})$. The destination $\mathrm{D}$ may fall within the communication range of a carrier and receive a copy of the message-carrying molecule that $S$ transmitted as in (D).

Previous efforts in epidemic information dissemination for molecular communication include collision-based molecular 
(A)

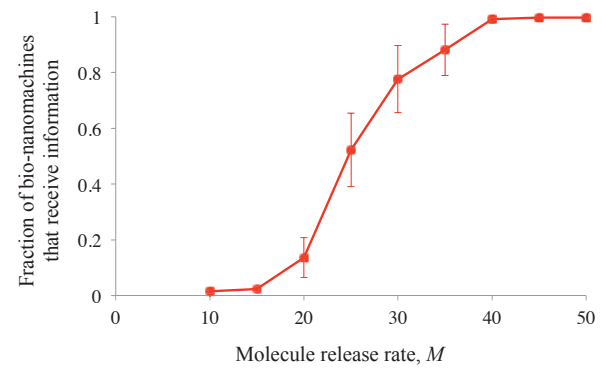

(C)

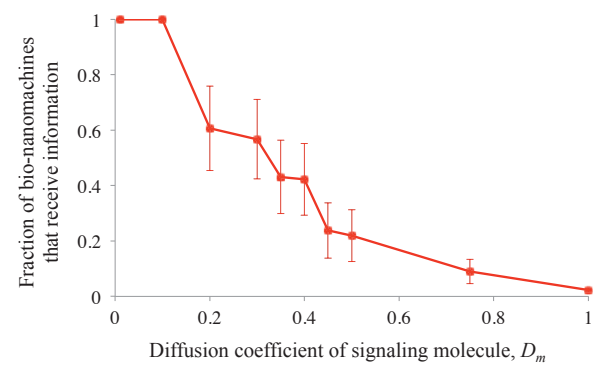

(B)

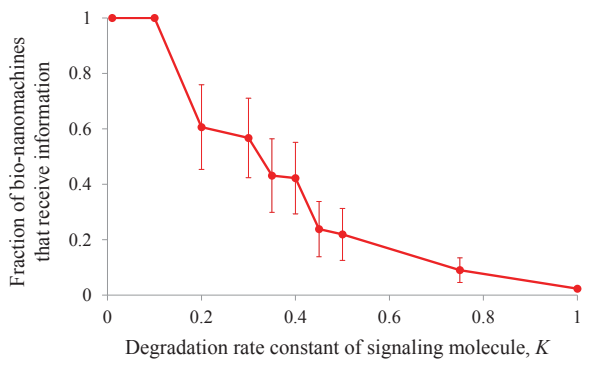

(D)

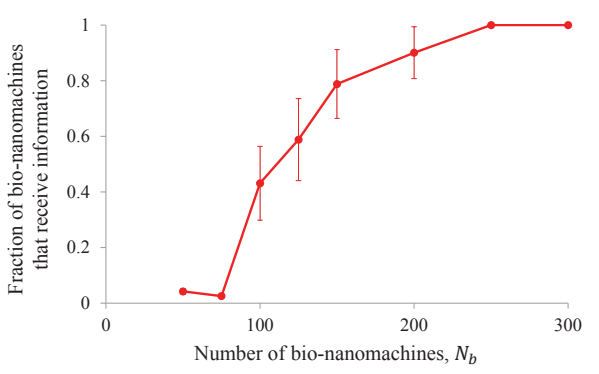

Figure 2: Impact of $M, K, D_{m}$ and $N_{b}$, on the fraction of bio-nanomachines that receive information

communication [4] in which mobile bio-nanomachines physically contact to relay messages among bio-nanomachines. Previous efforts also include opportunistic routing for molecular communication [2] and information spreading [3] in which bacteria functioning as carrier bio-nanomachines exchange DNA messages through conjugation processes. Other related efforts include diffusion-based molecular communication networks [1] in which static bio-nanomachines repeatedly relay information molecules released by source until destination is reached. Unlike these previous efforts, we consider the impact of molecule diffusion in epidemic information dissemination in the context of mobile molecular communication networks [6]. Furthermore, we demonstrate an application of the epidemic information dissemination method to targeted drug delivery.

\section{THE MODEL}

The number $N_{b}$ of mobile carriers (bio-nanomachines) are used to expand the communication range of a source (bionanomachine) in an $L \times L$ two-dimensional environment. A source transmits a message-carrying molecule. (A source may keep transmitting message-carrying molecules.) Carriers move randomly in the environment with the diffusion coefficient of $D_{b}$. Carriers get infected when the concentration of the molecule transmitted by the source exceeds a threshold concentration $H$. Infected carriers synthesize the same type of molecule that the source transmitted and release them into the environment at the rate $M$ for an infected period of $T_{R}$. After $T_{R}$, they enter the immune period for $T_{I}$. In the immune period, carriers continue to move randomly in the environment. After the immune period, carriers become reactive again to the concentration of the molecule. A destination (bio-nanomachine) may eventually receive the information-carrying molecule (namely, become infected).
The molecules diffuse with the diffusion coefficient $D_{m}$ and degrade with the degradation rate constant $K$ according to the following partial differential equation:

$$
\frac{\partial C}{\partial t}=D_{m} \Delta C-K C,
$$

where $C$ denotes the concentration of the molecule.

\section{PERFORMANCE EVALUATION}

We examine the impact of model parameters $\left(M, K, D_{m}\right.$ and $N_{b}$ ) on the performance of the epidemic information dissemination method. In performance evaluation, we assume that one bio-nanomachine is a source, and all other bio-nanomachines are carriers as well as destinations. All these bio-nanomachines are mobile and move randomly in the environment. For a performance metric, we use the mean fraction of bio-nanomachines that successfully receive information (or become infected) during 100 hours of a simulation run. By default, $L=10(\mathrm{~mm}), N_{b}=100$ (including one source and carriers/destinations), $H=0.03\left(1 / \mathrm{mm}^{3}\right)$, $M=25$ (1/hour), $D_{m}=0.36\left(\mathrm{~mm}^{2} /\right.$ hour $)$ from the diffusion coefficient of a protein molecule, $K=0.01$ ( $1 /$ hour), $D_{b}=1.79\left(\mathrm{~mm}^{2} /\right.$ hour$)$ from the diffusion coefficient of a bacterium, $T_{R}=0.035$ (hours) and $T_{I}=14$ (hours). At the begging of a simulation run, the source is placed at the center of the environment and other bio-nanomachines at randomly selected locations in the environment.

Simulation results are shown in Fig. 2 and summarized below:

- The fraction of bio-nanomachines that receive information increases as $M$ increases (Fig. 2A) or $k$ decreases (Fig. 2B) as expected.

- The fraction of bio-nanomachines that receive informa- 
tion decreases as $D_{m}$ increases (Fig. 2C). This is because, as $D_{m}$ increases, molecules diffuse more quickly and remain in the environment for a shorter period of time. This makes it difficult for bio-nanomachines to receive information.

- The fraction of bio-nanomachines that receive information increases as $N_{b}$ increases (Fig. 2D). This is because, as $N_{b}$ increases, the distance between bionanomachines becomes shorter, which increases the probability that bio-nanomachines receive information from information-carrying bio-nanomachines.

\section{APPLICATION TO TARGET DETECTION}

We consider a target detection application of mobile molecular communication networks [7]. Target detection is a functionality of mobile molecular communication networks to detect a target in a given environment. In nanomedical applications, targets can be disease sites, pathogens, infectious micro-organisms, or biochemical weapons that represent a potential threat to the environment; the timely detection of targets are important to provide immediate treatments or further analysis of the environment. The application goal considered in this section is to gather bio-nanomachines around targets for drug delivery or imaging purposes. In the following, we first describe a model of mobile molecular communication networks proposed in previous work. We then extend the model with the epidemic information dissemination method described in this paper and show the potential of the extended model in the context of targeted drug delivery.

\subsection{Leader-Follower-Based Model}

In our previous work [5], we designed a mobile molecular communication network according to the leader-follower based model (LF model) for targeted drug delivery applications. The LF model assumes two types of mobile bionanomachine, leader and follower bio-nanomachines that cooperate to control the spatial distribution of bio-nanomachines in a self-organized manner:

- Leader bio-nanomachines distribute in the environment to detect a target. Upon detecting a target, leader bio-nanomachines start releasing attractant molecules while they continue to move in the environment. The attractant molecules may form an attractant gradient in such a manner that the concentration is the highest around the target and decreases with distance from the target.

- Follower bio-nanomachines move in the environment and detect an attractant molecule. In the presence of attractant molecules, follower bio-nanomachines move preferentially to the higher attractant concentration. Follower bio-nanomachines implement application dependent functionalities such as release drug molecules at target locations.

\subsection{Leader-Follower-Amplifier-Based Model}

In this paper, we extend the LF model based on the epidemic information dissemination method. In the extended model, referred to as the leader-follower-amplifier based model (LFA model), we incorporate amplifier bio-nanomachines

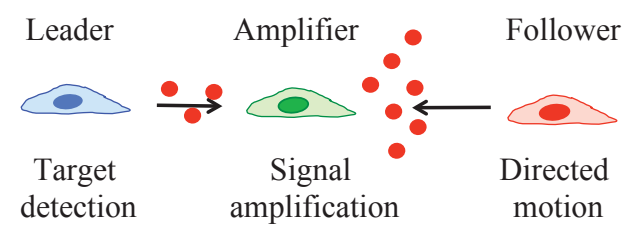

Figure 3: Leader-follower-amplifier (LFA) model

that amplify the attractant concentration by using the epidemic information dissemination method described in this paper. Here leader, follower, and amplifier bio-nanomachines correspond with sources, destinations, and carriers in Fig. 1, respectively. In the LFA model, leader and follower bionanomachines behave in the same manner as in the LF model. Amplifier bio-nanomachines move in the environment and detect an attractant molecule. Upon detecting an attractant molecule, amplifier bio-nanomachines start releasing attractant molecules (Fig. 3). In this way, amplifier bio-nanomachines help leader bio-nanomachines form an effective concentration gradient to attract follower bionanomachines.

\subsection{Simulation Results}

We conduct simulation experiments to demonstrate the potential of the LFA model. The environment is $10(\mathrm{~mm})$ $\times 10(\mathrm{~mm})$, containing a $0.1(\mathrm{~mm}) \times 0.1(\mathrm{~mm})$ target at the center of the environment. At the boundaries of the environment, bio-nanomachines bounce back while attractant molecules are absorbed and removed. The number of bio-nanomachines used in simulations are 50 for leaders, 50 for amplifiers and 100 for followers. Other parameter values are the following: $H=0.03\left(1 / \mathrm{mm}^{3}\right), M=25(1 /$ hour $)$, $D_{m}=0.36\left(\mathrm{~mm}^{2} /\right.$ hour $), K=0.01$ (1/hour), $D_{b}=1.79$ $\left(\mathrm{mm}^{2} /\right.$ hour $), T_{R}=0.035$ (hours) for leaders, $T_{R}=0.07$ (hours) for amplifiers, and $T_{I}=14$ (hours).

Fig. 4A shows how the mean distance between a bionanomachine and the target changes over time in the LF and LFA models. As shown in the figure, the LFA model successfully allows bio-nanomachines to move closer to the target. Fig. 4B shows the spatio-temporal distribution of bio-nanomachines and the attractant concentration in the LF and LFA models. As shown in the figure, the attractant concentration in the LF model is high in a limited area around the target, while in the LFA model the attractant concentration spreads over a wider area and attracting the more number of follower bio-nanomachines toward the target.

\section{CONCLUSION}

This paper proposed an information dissemination method for molecular communication among mobile bio-nanomachines. A simplistic model of the proposed method was presented and numerical results were shown to evaluate the performance in terms of the fraction of bio-nanomachines that receive information. Further, application to the target detection problem in mobile molecular communication was demonstrated. Future research is to fully evaluate the performance of the proposed method, develop a more realistic model by considering the physical and chemical nature of the proposed method, and conduct wet-laboratory experiments 

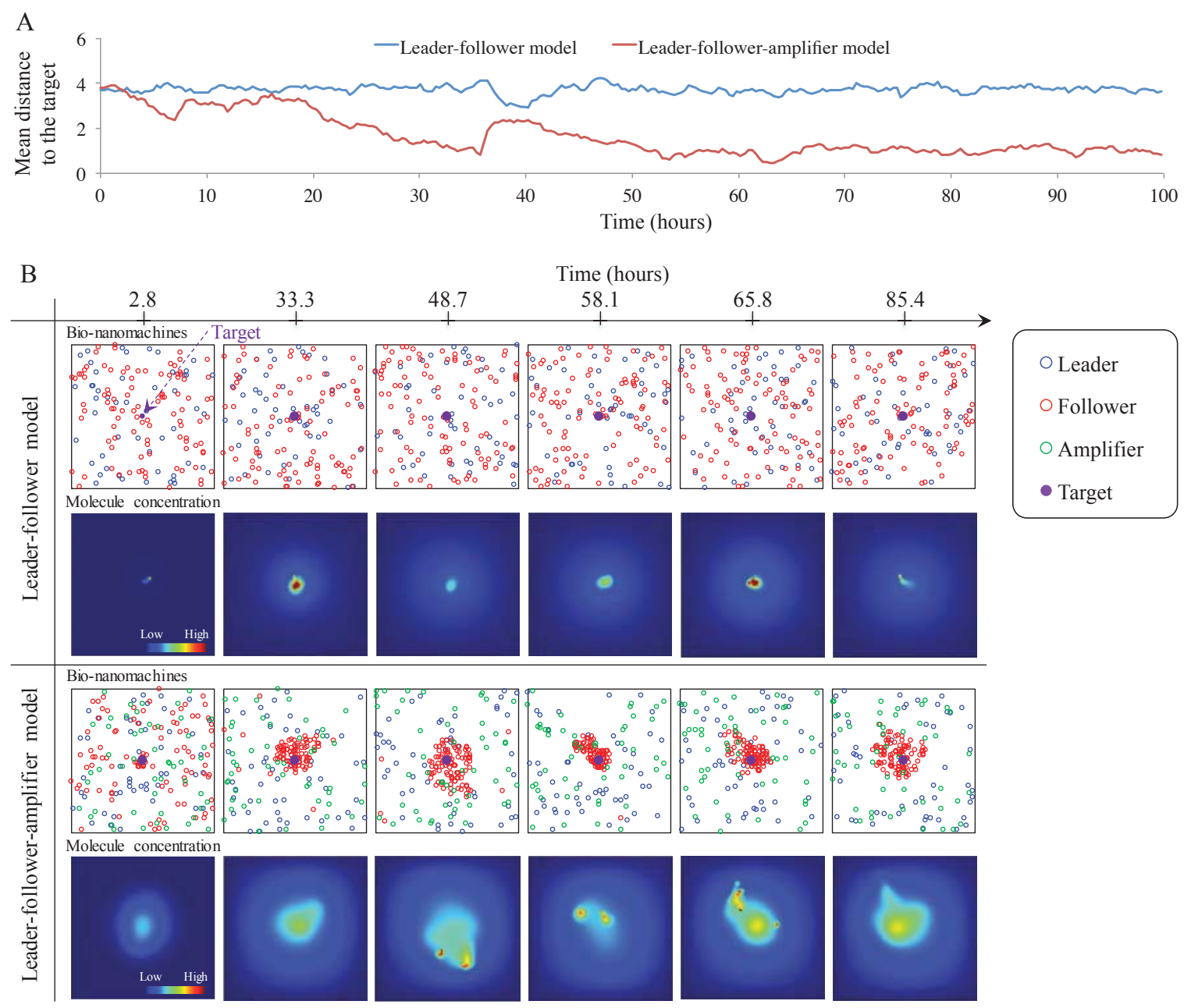

Figure 4: A. Mean distance from a follower bio-nanomachine to the target. B. Spatio-temporal distributions of bio-nanomachines and attractant concentration in the LF and LFA models.

to demonstrate the implementability.

\section{REFERENCES}

[1] A. Aijaz. Opportunistic routing in diffusion-based molecular nanonetworks. IEEE Wireless Communications Letters, 4(3):321-324, June 2015.

[2] S. Balasubramaniam and P. Lio. Multi-hop conjugation based bacteria nanonetworks. IEEE Transactions on Nanobioscience, 12(1):47-59, 2013.

[3] G. Castorina, L. Galluccio, and S. Palazzo. On modeling information spreading in bacterial nano-networks based on plasmid conjugation. IEEE Transactions on NanoBioscience, 15(6):567-575, 2016

[4] A. Guney, B. Atakan, and O. B. Akan. Mobile ad hoc nanonetworks with collision-based molecular communication. IEEE Transactions on Mobile Computing, 11(3):353-366, March 2012.

[5] T. Nakano, Y. Okaie, S. Kobayashi, T. Koujin, C.-H. Chan, Y.-H. Hsu, T. Obuchi, T. Hara, Y. Hiraoka, and T. Haraguchi. Performance evaluation of leader-follower-based mobile molecular communication networks for target detection applications. IEEE Transactions on Communications, 2016.

[6] J. Namba, T. Nakano, Y. Okaie, and T. Hara. Epidemic information dissemination for molecular communication among mobile bio-nanomachines. In ACM International Conference on Nanoscale Computing and Communication, 2016.

[7] Y. Okaie, T. Nakano, T. Hara, and S. Nishio. Target Detection and Tracking by Bionanosensor Networks. Springer Briefs in Computer Science, 2016.

[8] Y. Okaie, T. Nakano, T. Hara, T. Obuchi, K. Hosoda, Y. Hiraoka, and S. Nishio. Cooperative target tracking by a bacterium-based mobile sensor network. IEEE Transactions on Nanobioscience, 13(3):267-277, 2014.

[9] A. Vahdat and D. Becker. Epidemic routing for partially-connected ad hoc networks. Technical Report CS-200006, Duke University, 2000.

[10] G. Wei, P. Bogdan, and R. Marculescu. Bumpy rides: Modeling the dynamics of chemotactic interacting bacteria. IEEE Journal of Selected Areas in Communication (JSAC), 31(12):879-890, 2013. 